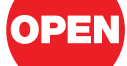

SUBJECT AREAS:

PHYSICS

OPTICS AND PHOTONICS

ULTRAFAST PHOTONICS

OPTICAL MATERIALS AND STRUCTURES

Received

27 July 2012

Accepted

10 October 2012

Published

9 November 2012

Correspondence and requests for materials should be addressed to G.G.G. Igurzadyan@ ntu.edu.sg) or T.Y. (Yuting@ntu.edu.sg)

\section{The Origin of Fluorescence from Graphene Oxide}

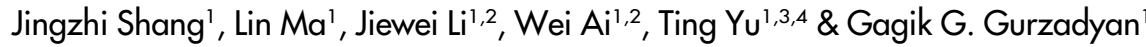

'Division of Physics and Applied Physics, School of Physical and Mathematical Sciences, Nanyang Technological University, Singapore 637371, ${ }^{2}$ Key Laboratory for Organic Electronics \& Information Displays (KLOEID) and Institute of Advanced Materials (IAM), Nanjing University of Posts and Telecommunications, Nanjing 210046, China, ${ }^{3}$ Department of Physics, Faculty of Science, National University of Singapore, Singapore 1 17542, ${ }^{4}$ Graphene Research Centre, National University of Singapore, 2 Science Drive 3, Singapore 117542.

Time-resolved fluorescence measurements of graphene oxide in water show multiexponential decay kinetics ranging from 1 ps to 2 ns. Electron-hole recombination from the bottom of the conduction band and nearby localized states to wide-range valance band is suggested as origin of the fluorescence. Excitation wavelength dependence of the fluorescence was caused by relative intensity changes of few emission species. By introducing the molecular orbital concept, the dominant fluorescence was found to originate from the electronic transitions among/between the non-oxidized carbon regions and the boundary of oxidized carbon atom regions, where all three kinds of functionalized groups $\mathrm{C}-\mathrm{O}, \mathrm{C}=\mathrm{O}$ and $\mathrm{O}=\mathrm{C}-\mathrm{OH}$ were participating. In the visible spectral range, the ultrafast fluorescence of graphene oxide was observed for the first time.

ollowing the exciting research of graphene $e^{1-3}$, chemically derived graphene oxide (GO) and reduced GO
(rGO) nowadays have attracted great interest due to the candidate status instead of graphene in some
aspects, facile synthesis and promising applications ${ }^{4-7}$. GO and rGO supercapacitor composites ${ }^{8}$, permeation and conducting thin films $s^{7,9}$ have been realized and the tunable optical characteristics have also raised much attention from light-emitting, biosensing and nonlinear optics communities ${ }^{10}$. For the as-prepared GO with the typical C:O ratio $(2-4)^{6}$, the widespread fluorescence has been reported from visible to near infrared (NIR) range and the maximum intensity is located between 500 and $800 \mathrm{~nm}^{6,10-15}$. For rGO, blueshift ${ }^{11,16-18}$ and redshift ${ }^{12}$ of the fluorescence from UV to NIR have been observed. Luo et al $^{12}$ proposed that the bond distortions may contribute to the fluorescence of GO and rGO. Chien and Eda et $a l^{11,16}$ have pointed out the disorder-induced states and the newly formed graphitic domains of sp2 clusters during reduction are responsible for GO photoluminescence (PL) and rGO blue PL, respectively. Galande et $a l^{19}$ have studied the $\mathrm{pH}$-dependent fluorescence of GO and suggested the emission of quasi-molecular fluorophores accounted for this behavior. Besides, Gokus et al ${ }^{20}$ have observed the similar PL features in oxygen plasma treated graphene compared to that from GO and assigned it to COrelated localized electronic states of oxidation sites. Till now, although much progress has been achieved in GOrelated studies, the origin of the fluorescence of GO is still a controversial issue.

In this work, we focus on the fluorescence of as prepared GO in water. Steady state and time-resolved fluorescence (TRF) measurements have been conducted in order to interpret the origin of GO emission. For the first time, TRF measurements with femtosecond resolution were performed. The GO model was built according to experimental data; the related electronic structures and properties were calculated based on density functional theory (DFT). Both experimental data and theoretical analysis suggest that the GO fluorescence is due to electron-hole recombination from conduction band $(\mathrm{CB})$ bottom and nearby localized electronic states to wide-range valance band (VB). In view of atomic structure, the GO emission is predominantly from the electron transitions among/between the non-oxidized carbon region $(-\mathrm{C}=\mathrm{C}-)$ and the boundary of oxidized carbon atom region $(\mathrm{C}-\mathrm{O}, \mathrm{C}=\mathrm{O}$ and $\mathrm{O}=\mathrm{C}-\mathrm{OH})$.

\section{Results}

XPS, XRD and Raman spectra. Figure 1a shows the C1s signal of the prepared graphite oxide powder obtained by $\mathrm{x}$-ray photoelectron spectrometer (XPS). This signal was fitted by four components: $\mathrm{C}=\mathrm{C} \& \mathrm{C}-\mathrm{C}(46.8 \%$, $284.6 \mathrm{eV}), \mathrm{C}-\mathrm{O}(43.1 \%, 286.7 \mathrm{eV}), \mathrm{C}=\mathrm{O}(5.0 \%, 288.0 \mathrm{eV})$ and $\mathrm{O}=\mathrm{C}-\mathrm{OH}(5.1 \%, 288.9 \mathrm{eV})$. The estimated $\mathrm{C} /$ $\mathrm{O}$ ratio is $\sim 2.4$. These features are consistent with the previous studies ${ }^{5,6,21,22}$. Figure $1 \mathrm{~b}$ presents the $\mathrm{X}$-ray diffraction (XRD) spectrum, where the main diffraction peak appears at $12.4^{\circ}$ with a full width at half 


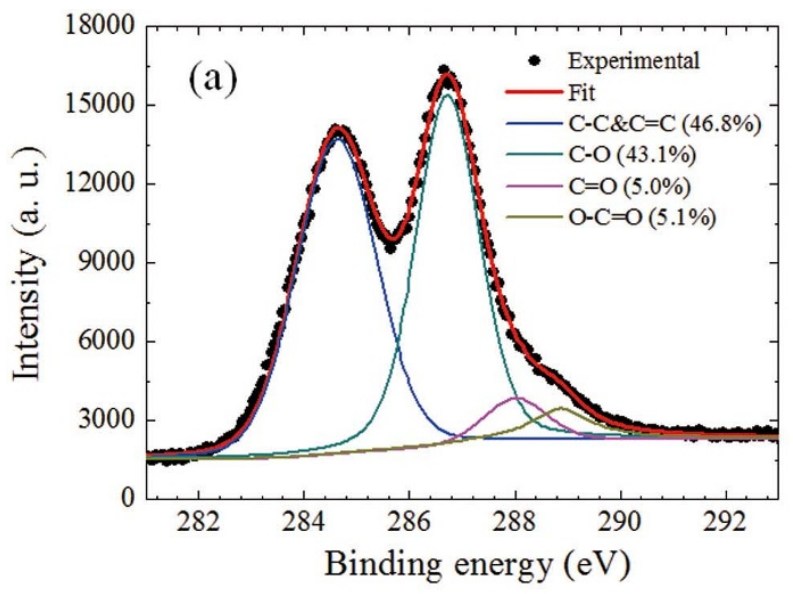

Figure $1 \mid$ (a) XPS, (b) XRD and (c) Raman spectra of graphite oxide.

maximum (FWHM) of $\sim 1.3^{\circ}$, being similar to other reports ${ }^{23,24}$. Correspondingly, the interlayer distance in graphite oxide is $\sim 0.71 \mathrm{~nm}$. The typical Raman spectrum of graphite oxide is shown in Figure 1c, where the D and G bands were observed clearly at $\sim 1350$ and $\sim 1598 \mathrm{~cm}^{-1}$ with the intensity ratio (or area ratio) $\mathrm{I}_{\mathrm{D}} / \mathrm{I}_{\mathrm{G}}$ of $\sim 1$ (or $\sim 1.76$ ), comparable with the previous observations ${ }^{23,25,26}$. The other three weak Raman bands, $2 \mathrm{D}, \mathrm{D}+\mathrm{G}$, and $2 \mathrm{G}$ appear at $\sim 2700, \sim 2925$ and $\sim 3156 \mathrm{~cm}^{-1}$, respectively.

Steady-state and transient absorption. Figure $2 \mathrm{a}$ shows the absorption spectrum of GO suspension in $2 \mathrm{~mm}$ cuvette ranging from 200 to $900 \mathrm{~nm}$. One main peak at $\sim 231 \mathrm{~nm}$ comes from the $\pi-\pi^{*}$ transition of $\mathrm{C}-\mathrm{C}$ and $\mathrm{C}=\mathrm{C}$ bonds in sp 2 hybrid regions and a shoulder peak at $\sim 303 \mathrm{~nm}$ is due to $n-\pi^{*}$ transition of the $\mathrm{C}=\mathrm{O}$ bond in sp3 hybrid regions $s^{14,27}$. In addition, there is no obvious band edge absorption feature found up to $900 \mathrm{~nm}$. Figure $2 \mathrm{~b}$ presents the transient absorption spectra in the visible range from 530 to $770 \mathrm{~nm}$. The positive absorbance change indicates the excited state absorption is dominant in the probing region. The small valley around $740 \mathrm{~nm}$ is caused by induced absorption of water since there is a water absorption band in this range ${ }^{28}$. Transient absorption signal at 560 to $760 \mathrm{~nm}$ decays multiexponentially (Figure S1); global fit gives four time constants: $2.1 \pm 0.2,13 \pm 2,90 \pm 10$ and $2000 \pm 200$ ps. The first three components correspond to the reported values in the previous pump-probe studies on $\mathrm{GO}^{29,30}$. The observed longest component is assigned to the carrier lifetime at the bottom of $\mathrm{CB}$, which is further confirmed by TRF measurements.
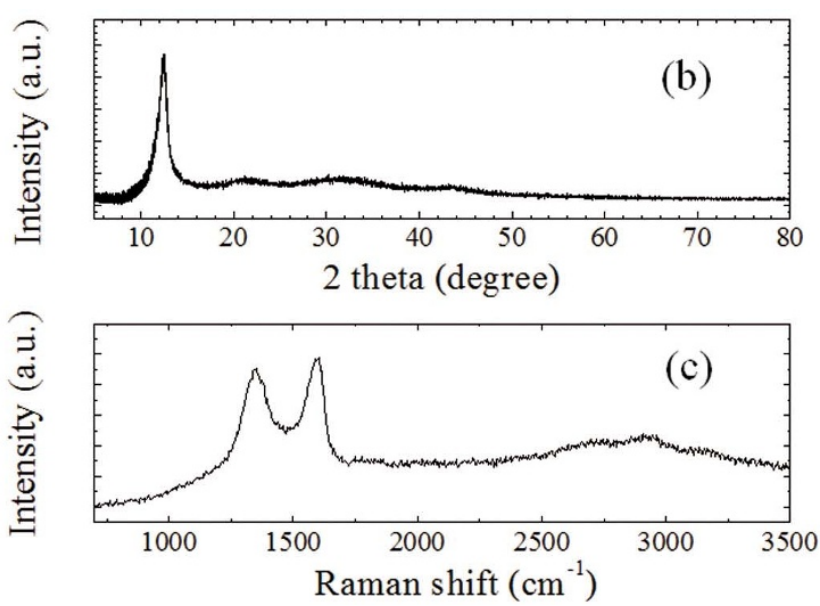

In Ref. 29, an ultrafast dynamics was reported by use of degenerate pump-probe $(800 \mathrm{~nm})$ spectroscopy. The turnover of kinetic curves within the first picosecond was observed with the increase of pump intensity. According to their proposed mechanism, the negative and positive transient absorption signals are due to the saturable absorption in sp2 regions and the two-photon absorption in sp3 regions, respectively. In particular, they mentioned that the optical transition for $800 \mathrm{~nm}$ in the sp3 domains is forbidden due to the large energy gap of sp3 domains. In other words, the electrons in the ground state of sp3 domains could not be effectively excited at low pump intensity. With the increase of pump intensity, the positive and negative transient absorption becomes comparable, thus, the turnover of kinetic curves was observed. However, the turnover of kinetic curves was not observed in our case and Ref 30 . The main reason is that we have used non-degenerate pump-probe technique with $\lambda_{\text {exc }}=480 \mathrm{~nm}$ (Ref. 30 used $\lambda_{\text {exc }}=400 \mathrm{~nm}$ ). Both sp 2 and sp3 domains are excited monophotonically with low pump intensity (comparable with case $\mathrm{C}$ on Fig. 3 of Ref.29). In the present work, the observed positive transient absorption spectra and kinetics are similar to those in Ref 30, where only positive transient absorption kinetics of GO and rGO were observed. Particularly, the longest lifetime component $\left(\tau_{3}+\tau_{4}\right)$ is larger than 300 ps for both $\mathrm{GO}$ and $\mathrm{rGO}^{30}$, which is consistent with our observations.

Fluorescence. Figure 3a shows the fluorescence excitation spectra for the emission wavelengths $\left(\lambda_{\text {em }}\right)$ from $350 \mathrm{~nm}$ to $650 \mathrm{~nm}$. It is clear that the excitation spectrum broadens with $\lambda_{\text {em }}$, and there is no
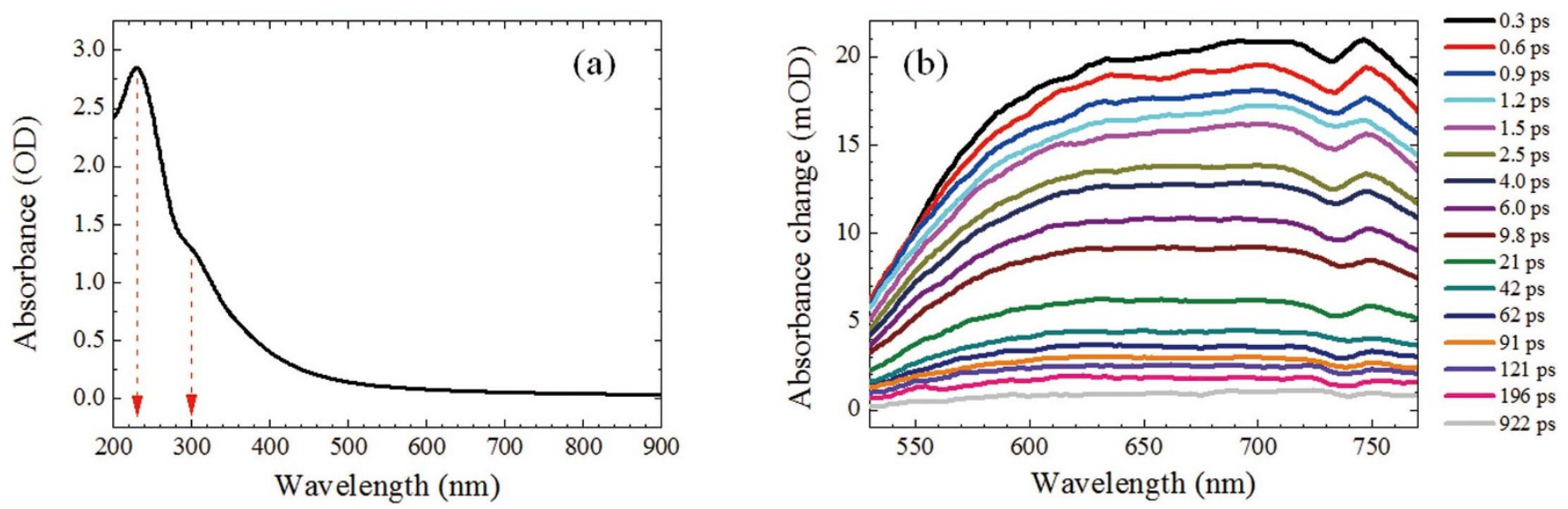

Figure $2 \mid$ (a) Steady state and (b) transient absorption spectra of GO in water. 

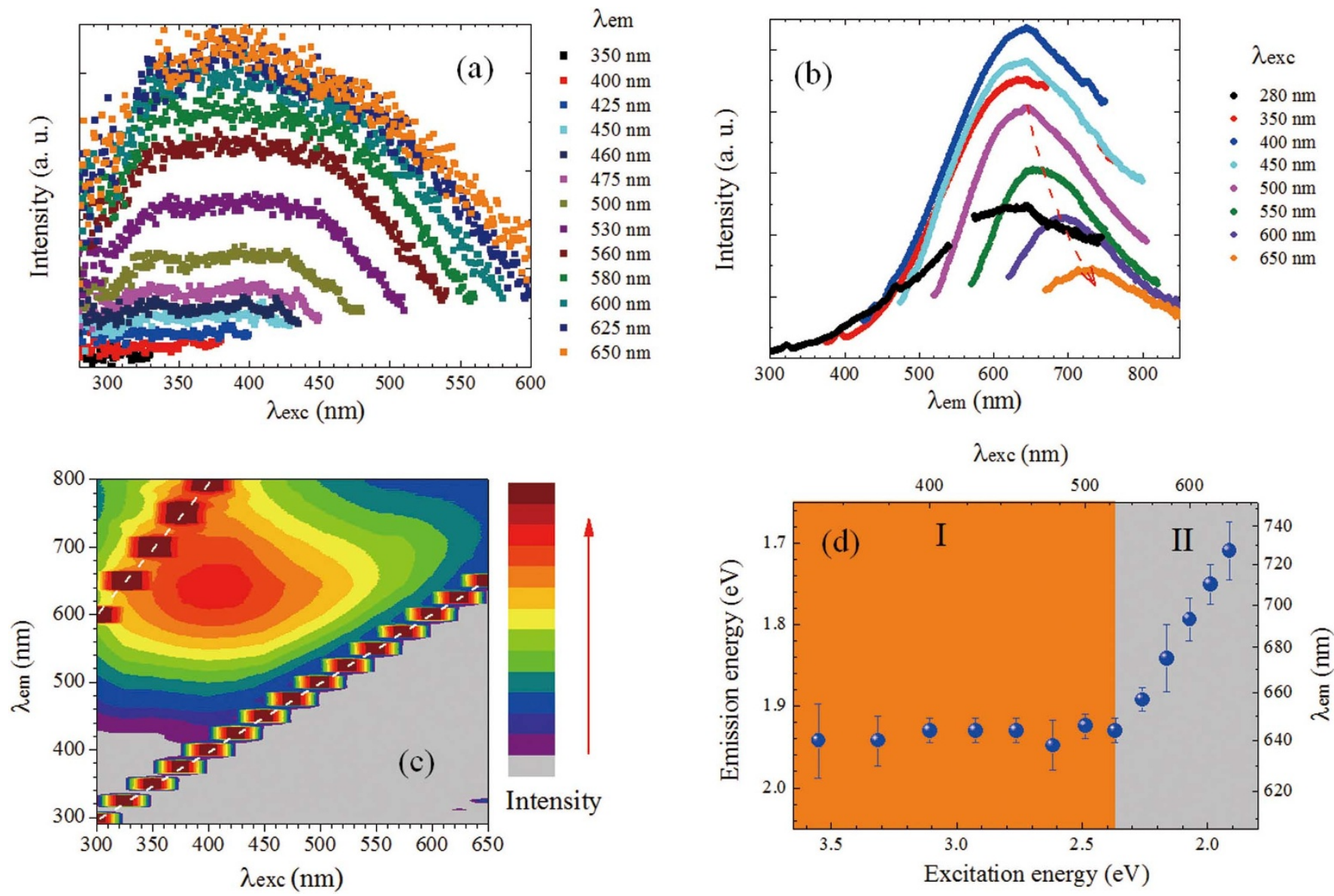

Figure $3 \mid$ Fluorescence excitation (a) and emission (b) spectra of GO in water; (c) fluorescence excitation-emission map of GO; two trains of strong signals indicated by dashed white lines are due to scattering of excitation light and its second order; (d) fluorescence emission energy/wavelength maxima versus excitation energy/wavelength.

obvious sharp absorption peak. The excitation light between $280 \mathrm{~nm}$ and $\lambda_{\mathrm{em}}$ all can be absorbed, which indicates the continuous absorption band exists in our sample. Moreover, our observation at $\lambda_{\mathrm{em}}=600 \mathrm{~nm}$ is well consistent with that measured at $\lambda_{\mathrm{em}}=$ $605 \mathrm{~nm}$ by others ${ }^{11}$. The clear structured fluorescence excitation spectra of GO at $\lambda_{\mathrm{em}}=511$ and $540 \mathrm{~nm}$ were observed at various $\mathrm{pH}$ values in Ref.19, which was not seen here. The diversity probably results from the different dielectric environment of GO. However, some of our broad excitation spectra show resemblance to those observed at $\lambda_{\text {em }}=665 \mathrm{~nm}^{19}$ and may contain similar spectral components. Figure $3 \mathrm{~b}$ shows the fluorescence emission spectra with excitation wavelength $\left(\lambda_{\text {exc }}=280-650 \mathrm{~nm}\right)$. The broad emission band appears between 400 and $800 \mathrm{~nm}$ analogous to previous reports on fluorescence spectra of $\mathrm{GO}^{6,10-14,17}$ and oxygenplasma treated graphene $e^{20}$. The maximum of the emission spectrum red-shifts versus $\lambda_{\text {exc }}$ (See Figure $3 c$ ), most pronounced in the excitation region II as shown in Figure 3d. In Region I (350-525 nm), the emission maximum is constant. On the whole, there is obvious dependence of both, excitation spectra versus $\lambda_{\text {em }}$ and emission spectra versus $\lambda_{\text {exc }}$. Such a behavior is indicative for multiple chromophore/fluorophore system ${ }^{31}$ involved with aromatic and oxidation groups.

Figures $4 \mathrm{a}-\mathrm{c}$ present the TRF decay curves of GO at $\lambda_{\text {exc }}=400 \mathrm{~nm}$ by time-correlated single photon counting (TCSPC) system. Similar measurements were also executed at $\lambda_{\operatorname{exc}}=480 \mathrm{~nm}$. The kinetic curves at $\lambda_{\mathrm{em}}=500-780 \mathrm{~nm}$ show a multiexponential decay feature. Four time constants are necessary to fit these curves and the corresponding fractional amplitudes are shown in Figure 4 d, respectively.
For $\lambda_{\text {exc }}=400$ or $480 \mathrm{~nm}$, the lifetimes are $\tau_{1}=30 \pm 15$ ps (or $30 \pm 15$ ps); $\tau_{2}=140 \pm 10$ ps (or $145 \pm 10$ ps); $\tau_{3}=510 \pm 20$ ps (or $540 \pm 20 \mathrm{ps}$ ); $\tau_{4}=1680 \pm 50$ ps (or $1780 \pm 60 \mathrm{ps}$ ). There is no obvious dependence of lifetime on $\lambda_{\text {exc }}$ found for these two excitation cases. Recently, three emission lifetimes for GO: 200, 500, 1400 ps have been extracted ${ }^{11}$, which well agree with obtained $\tau_{2}, \tau_{3}$ and $\tau_{4}$ in the present study. In addition, similar multiexponential decay (40, 250 and 1000 ps) was also found in the oxygen-plasma treated graphene $e^{20}$. Note that, the longest component from fluorescence decay is comparable to the longest carrier lifetime (2000 ps) obtained from transient absorption data, which supports that emission corresponding to $\tau_{4}$ originates from the bottom of CB. As shown in Figure $4 \mathrm{~d}$, with the increase of $\lambda_{\text {em }}$ from 500 to $700 \mathrm{~nm}$, the amplitude of $\tau_{1}$ decreases while the other three increase gradually. At $\lambda_{\mathrm{em}}>700 \mathrm{~nm}$, the amplitudes of all components maintain. The small fluctuation around $740 \mathrm{~nm}$ is related to the absorption of water, as indicated above.

Furthermore, by use of the emission spectra at $\lambda_{\text {exc }}$ of 400 and $475 \mathrm{~nm}$ (Figure $3 \mathrm{~b}$ ) and the fractional amplitudes obtained from TRF measurements (Figure $4 \mathrm{~d}$ ), the contribution of four components was extracted, as shown in Figure 5. As expected, the peaks from $\tau_{2}, \tau_{3}$ and $\tau_{4}$ are dominant for $\lambda_{\mathrm{em}}>600 \mathrm{~nm}$ and the peak from $\tau_{1}$ plays important role in the shorter wavelength region (500$600 \mathrm{~nm})$. For both cases, the peak positions of $\tau_{2}, \tau_{3}$ and $\tau_{4}$ are comparable. It is also noted that the emission peaks blue shift with the decrease in lifetime. In other words, these observations indicate that the emission lifetime decreases from the lower to higher excited states. Observed rapid red-shift of $\lambda_{\text {em }}$ maximum versus $\lambda_{\text {exc }}$ (region 

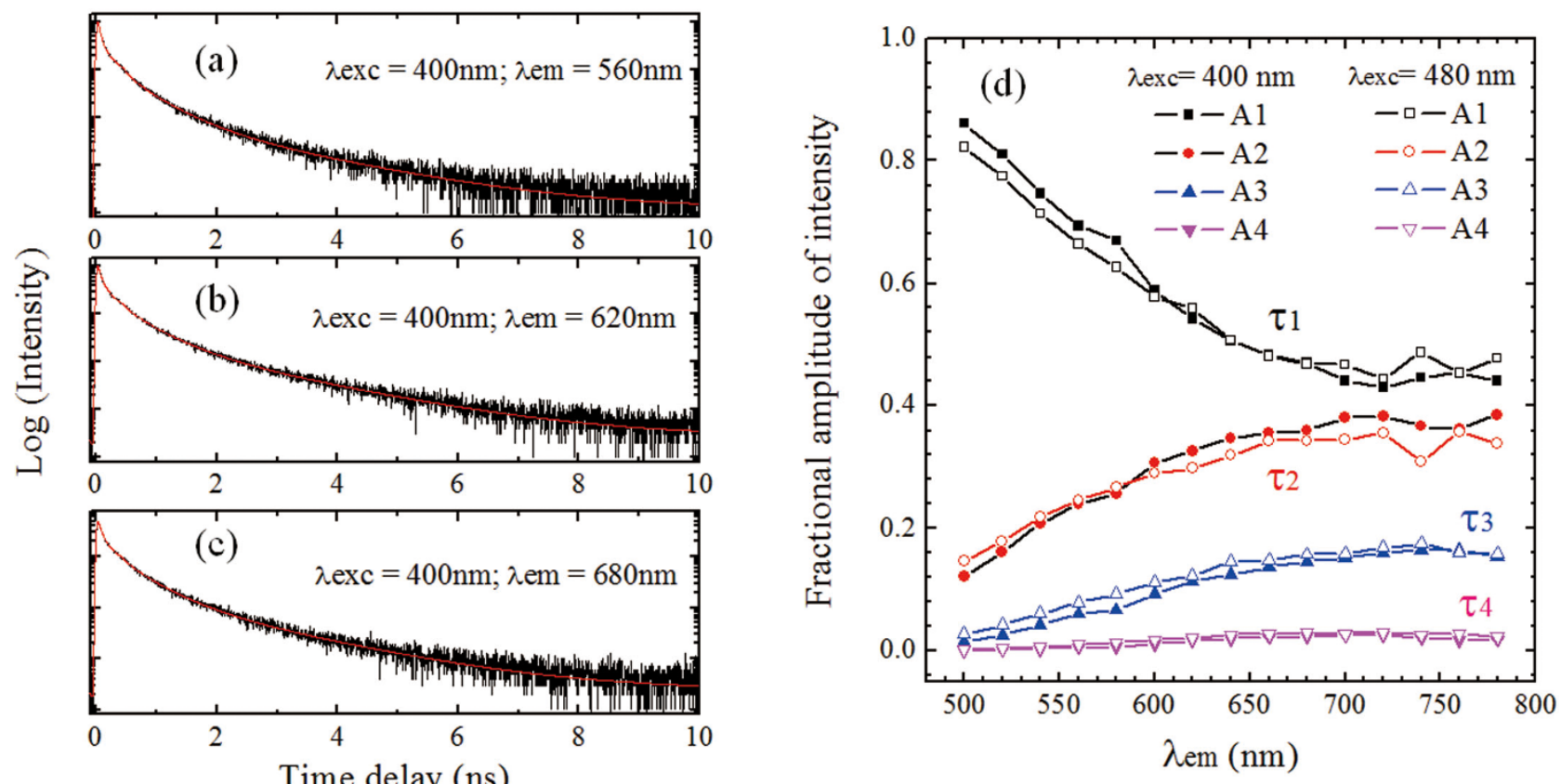

Figure $4 \mid(a-c)$ TCSPC measurements of GO in water at $\lambda_{\text {exc }}=400 \mathrm{~nm}$; (d) fractional amplitudes of four time components obtained by TCSPC measurements at $\lambda_{\text {exc }}=400$ and $480 \mathrm{~nm}$.

II in Figure 3d) can be explained in terms of stronger contribution (fractional intensities) of longer time components of emission to the steady-state fluorescence (Figure $3 \mathrm{~b}$ ).

Figures $6 \mathrm{a}-\mathrm{f}$ show the femtosecond fluorescence upconversion data for GO suspensions at $\lambda_{\mathrm{exc}}=400 \mathrm{~nm}$ and $\lambda_{\mathrm{em}}=540-660 \mathrm{~nm}$. In the first $50 \mathrm{ps}$, there are mainly two decay components: $\tau_{0}=0.9 \pm$ $0.2 \mathrm{ps} ; \tau_{1}=26 \pm 3 \mathrm{ps}$. The relative fractional amplitudes vary with $\lambda_{\mathrm{em}}$ (Figure $6 \mathrm{~g}$ ), and the contribution of $\tau_{1}$ increases with $\lambda_{\mathrm{em}}$. Decay lifetime $\tau_{1}$ corresponds well to the shortest component measured with TCSPC. Our up-conversion measurements indicate that another ultrafast emission state exists in the GO sample, which is responsible for $\tau_{0}$ decay. The origin of the ultrafast component is attributed to the higher excited state emission (see below).

DFT calculations. Figure 7a shows top (upper panel) and side (lower panel) views of the theoretical atomic structural model of GO cell, which is built based on the experimental data, mainly XPS and absorption spectra. In the following calculations, the periodic structure made of large amount of GO cells was introduced, which is closer to the real structure compared with the single cell. By adjusting the relative positions of these bonds, the absorption spectra (Figure S2) and absorption wavelengths (Figure S3) were calculated, which are in agreement with the experimental data (Figure 2a). It indicates the positions of $\mathrm{C}-\mathrm{C}, \mathrm{C}-\mathrm{O}, \mathrm{C}=\mathrm{O}$ and $\mathrm{O}=\mathrm{C}-\mathrm{OH}$ in sample are well reproduced in the model.

Previously, the theoretical calculation $\mathrm{s}^{32,33}$ on oxidized graphene suggested a band gap ranging from 0 to few $\mathrm{eV}$, which depends on the ratio of carbon bonds to oxidation functional groups and their different arrangements. By estimating the typical sp2 cluster size $(2.5 \sim 6 \mathrm{~nm})^{16,26}$, the predicted band gap in GO is from 0.58 to $0.24 \mathrm{eV}^{6}$. Experimentally, a $0.25 \mathrm{eV}$ band gap of oxidized graphene was determined by use of scanning probe microscopy ${ }^{34}$. Bansal et al have directly observed the density of states near Fermi level of GO by capacitive photocurrent spectroscopy $y^{35}$. In our case, the electronic band structure (left panel) and density of states (DOS: right panel) of GO were calculated as shown in Figure $7 \mathrm{~b}$. The band gap of this GO
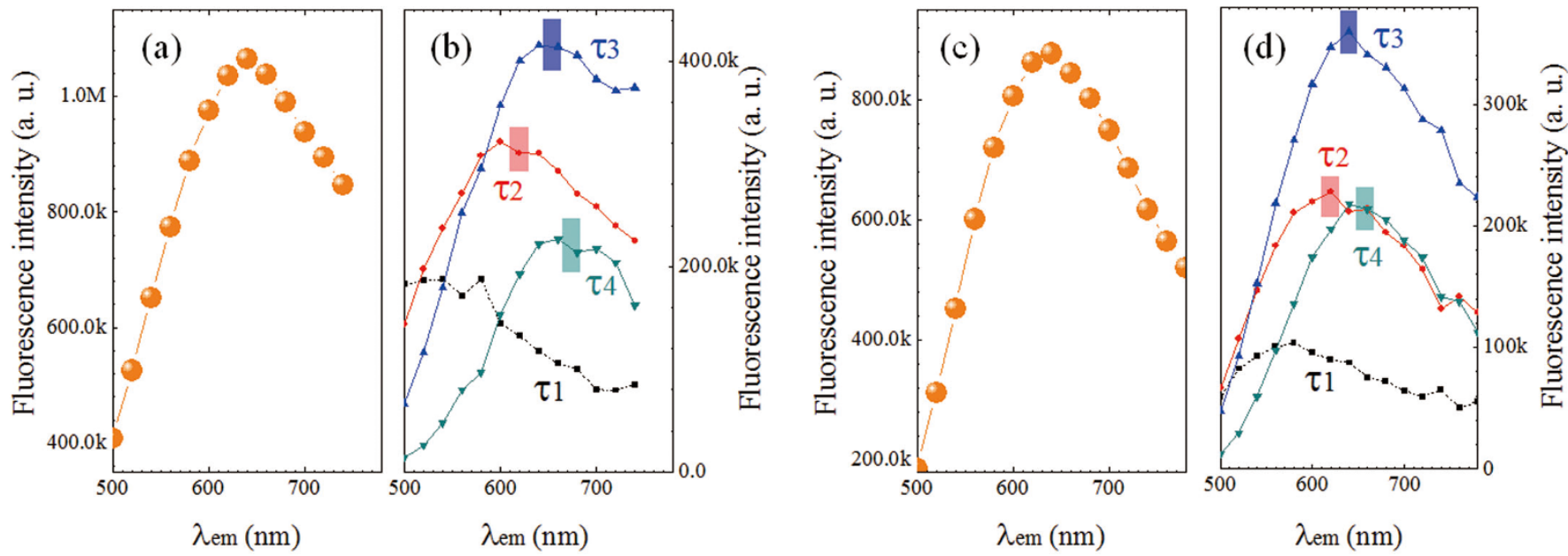

Figure $5 \mid$ Fluorescence emission spectra at (a) $\lambda_{\text {exc }}=400 \mathrm{~nm}$ and (c) $\lambda_{\text {exc }}=480 \mathrm{~nm}$, and the corresponding spectra (b) and (d) of four time components as denoted by square $\left(\tau_{1}\right)$, circle $\left(\tau_{2}\right)$, upward $\left(\tau_{3}\right)$ and downward $\left(\tau_{4}\right)$ triangles, respectively. 

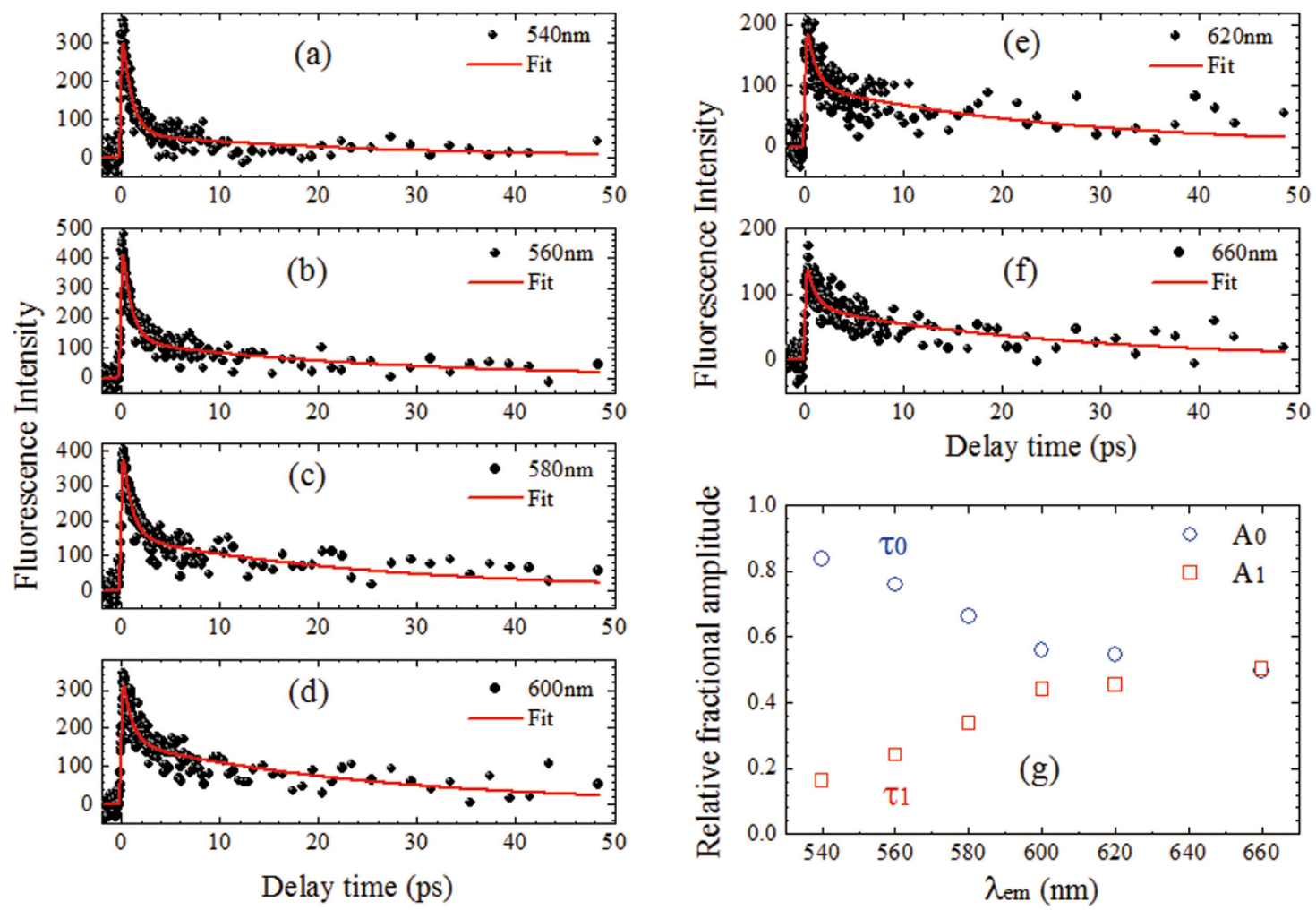

Figure $6 \mid(\mathrm{a}-\mathrm{f})$ Fluorescence upconversion kinetics at $\lambda_{\mathrm{exc}}=400 \mathrm{~nm}$ and $\lambda_{\mathrm{em}}=540-660 \mathrm{~nm}$; (g) relative fractional amplitudes of two lifetime components $\left(\tau_{0}=0.9 \pm 0.2 \mathrm{ps} ; \tau_{1}=26 \pm 3 \mathrm{ps}\right)$ versus wavelength.

structure is $\sim 0.6 \mathrm{eV}$, which implies that the band edge-related absorption peak may appear in the infrared range. Meanwhile, the calculation shows a band edge transition at $1987 \mathrm{~nm}$ (Figure S3). Indeed, an absorption band of GO film on quartz was observed at $\sim 1914 \mathrm{~nm}$ in the near IR region as shown in Figure S4, which further supports the validity of the used model. Moreover, it is noted that there are some relative flat bands nearby the bottom of $\mathrm{CB}$, where the localized electronic states exist. For the dominant excited state absorption (Figure 2b), the corresponding transitions are from the $\mathrm{CB}$ bottom and nearby localized states to the higher excited states. For the observed fluorescence of $\lambda_{\mathrm{em}}=400-800 \mathrm{~nm}$, the origin is attributed to the electronic transitions from the bottom of $\mathrm{CB}$ and the nearby localized states to the VB as shown in Figure 7c. The multiple exponential photoluminescence decay was caused by different emission states of band edge $\left(\tau_{4}\right)$ and localized electronic states $\left(\tau_{0^{-}} \tau_{3}\right)$ in CB. Furthermore, as indicated by red dots in Figure $7 \mathrm{~b}$, the lowest three bands of $\mathrm{CB}$ contain five localized states. Therefore, observed five fluorescence decay times at $\lambda_{\text {em }}=540-660 \mathrm{~nm}$ will originate from these states. Moreover, the lowest two bands in CB mainly contribute to the steady-state emission at $\lambda_{\mathrm{em}}>600 \mathrm{~nm}$ (Figure 5).

Physically, these states originate from the Anderson localization ${ }^{36-38}$ of electronic wavefunctions in the heterogeneous and disordered sp $2 /$ sp 3 complex structure. As shown in Figure $7 \mathrm{~b}$, the energy bands near theses localized states are flat, which indicates the electron effective masses at these regions are close to infinity. Thus, the excited electrons in these flat bands are confined in the certain momentum space. These electrons will relax radiatively (interband transition) rather than non-radiatively (intraband relaxation). Previously, the localization of electronic wavefunctions in disordered graphene ${ }^{39}$, chemically modified graphene $e^{40}$ and graphene nanoribbons ${ }^{41}$ has been studied theoretically. The existence of localized states in disordered graphene nanoribbons was also proved experimentally $^{42}$. Structurally, these localized states are probably from few kinds of chromophone/fluorophore structures consist of aromatic and oxidation groups in GO and not the states caused by random disorder in GO. It is supported by measurements of other GO samples with different proportions of functional groups (Figure S7-S15).

Molecular-like features (the $\mathrm{pH}$-dependent visible fluorescence) of GO have recently been revealed by the steady state fluorescence measurements ${ }^{19}$, where the emission is assigned to quasi-molecular fluorophores consisting of $\mathrm{O}=\mathrm{C}-\mathrm{OH}$ groups and neighboring carbon atoms. In order to check this viewpoint, the GO has been treated as a large-size two dimensional molecule. The orbital energy levels of this structure were calculated (Figure 7d) and the corresponding electronic orbitals are presented in Figure 7e. The energy differences between HOMO and LUMO are about $0.6 \mathrm{eV}$ and the non-oxidized $\mathrm{C}-\mathrm{C} \& \mathrm{C}=\mathrm{C}$ regions take main responsibility for their formation. Obviously, the observed fluorescence of GO between 400 and $800 \mathrm{~nm}$ is not from LUMO to HOMO electronic transition. Recently, Itoh has reviewed the fluorescence from higher excited states of organic molecules ${ }^{43}$, such as azulenes, aromatic acenes and aromatic carbonyl compounds. In GO sample, large amount of analogues of aromatic acenes, o-hydroxybenzaldehyde and other fluorescent molecules exist. Therefore, the fluorescence from higher excited states should be taken into account. For the emission near $640 \mathrm{~nm}, \mathrm{LUMO}, \mathrm{L}+1, \mathrm{~L}+2, \mathrm{HOMO}$ and $\mathrm{H}-1$ to $\mathrm{H}-5$ could be responsible, where the corresponding atom groups include $-\mathrm{C}=\mathrm{C}$ with $-\mathrm{C}-\mathrm{O},-\mathrm{C}=\mathrm{O}$, and $\mathrm{O}=\mathrm{C}-\mathrm{OH}$. As seen in Figure $7 \mathrm{e}, \mathrm{L}+1, \mathrm{~L}+2$, and $\mathrm{H}-1$ to $\mathrm{H}-5$ mainly come from the regions containing both $\mathrm{C}=\mathrm{C}$ - and oxidized groups. Particularly, for the dominant yellow (500-800 $\mathrm{nm}$ ) fluorescence of GO, the allowed electronic transitions are from LUMO to $\mathrm{H} 1-5$ and from $\mathrm{L}+1, \mathrm{~L}+2$ to HOMO. Moreover, the oxidized regions connected to non-oxidized $-\mathrm{C}=\mathrm{C}$ - more strongly contribute to the electronic orbitals than that from the inner part of oxidized regions. In other words, mainly the boundary of the oxidized regions that consists of oxidized carbon atoms and functionalized groups participates into the fluorescence emission rather than inner atoms from oxidized regions. Thus, the observed 
(a)
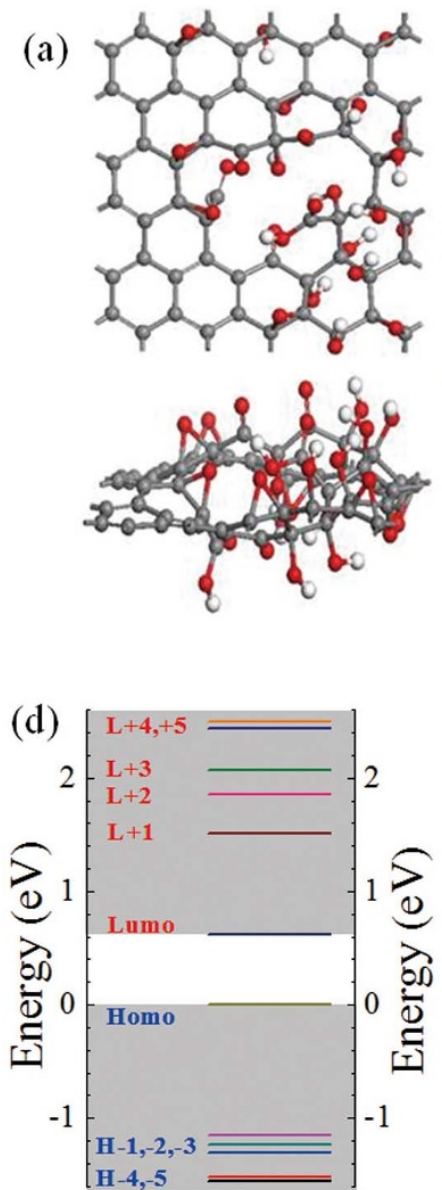
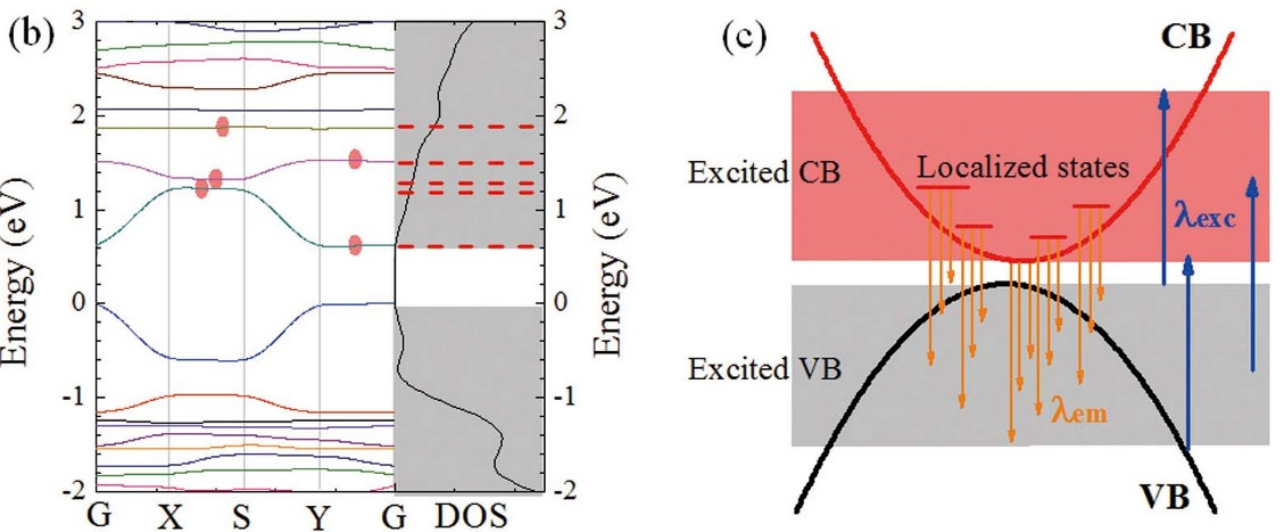

(e)
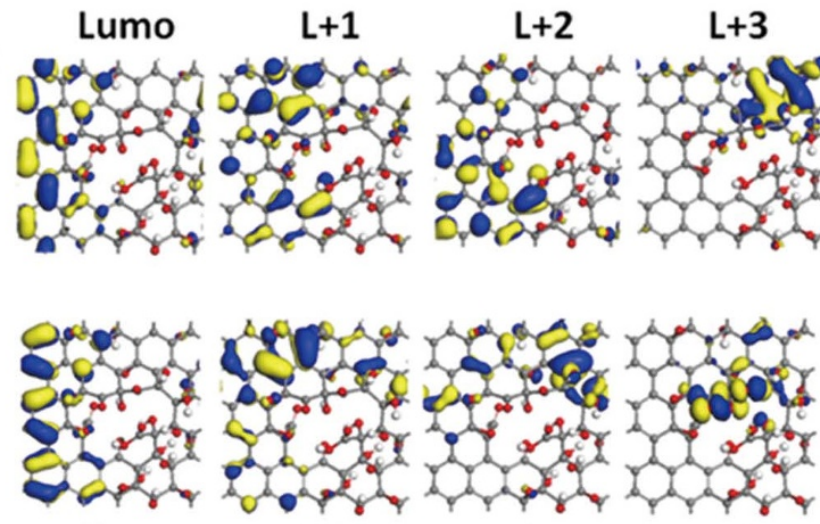

H-3

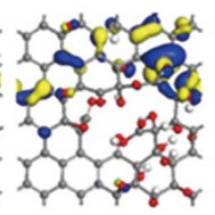

$\mathrm{H}-2$

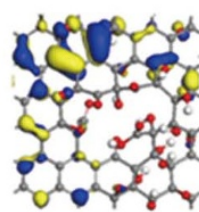

$\mathrm{H}-1$

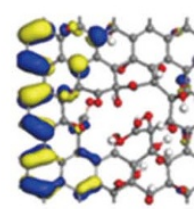

Homo
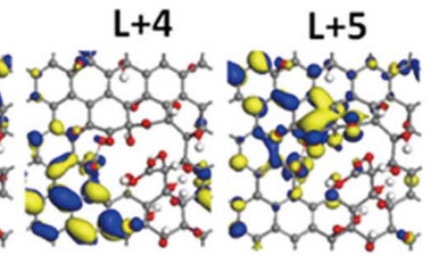

Figure 7 | (a) Top view and side view of GO model; (b) electronic band structure and density of states (DOS) of GO; (c) schematic of fluorescence excitation and emission of GO; (d) quasi-molecular orbital energy levels of GO; (e) fluorescence related orbitals of GO.

fluorescence mainly resulted from the electronic transitions among/ between the non-oxidized $-\mathrm{C}=\mathrm{C}$ - and the boundary of oxidized regions. Even though there is no direct experimental evidence for the precise role of three functional groups, we have observed that the fluorescence features of GO vary with different proportions of oxidized groups (Figures S10-S15). Our data indicates that the functionalized groups together with oxidized carbon atoms and nonoxidized $-\mathrm{C}=\mathrm{C}$ - form the dominant emission species in $\mathrm{GO}$.

\section{Discussion}

Previously, Chien and Eda et al suggested that the fluorescence of as prepared GO may originate from the optical transitions from structural disorder-induced localized states in the $\pi$ - $\pi^{*}$ gap of sp2 sites ${ }^{11,16}$. Luo $e t$ al also proposed that the fluorescence may be caused by bond disorder induced energy gaps rather than quantum confinement effect $^{12}$. The influence of functionalized groups on the fluorescence has not caused enough attention in these two groups ${ }^{11,12,16}$. Our studies show that the functionalized groups and some oxidized sp3 sites have important contribution to the fluorescence. Broad yellow fluorescence was also observed in $\mathrm{O}_{2}$ plasma-treated graphene by Gukus et $a^{20}$. By analyzing the fluorescence band broadening and timeresolved data, they suggested CO-related localized electronic state at oxidation sites being the fluorophores. It was noted that not sp2 sites emit fluorescence ${ }^{20}$. In our case, the sp2 sites are critical for the emission and the fluorescence band was found to consist of few components (emission species), as shown in Figure 5. Recently, Galande et al proposed that the GO fluorescence is from quasimolecular fluorophores formed by $\mathrm{COOH}$ groups and nearby sp2 carbon atoms according to steady state studies of $\mathrm{pH}$-dependent fluorescence ${ }^{19}$. We found all three kinds of functionalized groups $\mathrm{C}-\mathrm{O}, \mathrm{C}=\mathrm{O}$ and $\mathrm{O}=\mathrm{C}-\mathrm{OH}$ are related to the emission. In addition, the tunable fluorescence of $\mathrm{rGO}^{11,17,18}$ and graphene quantum dots ${ }^{4-46}$ has started to attract interest and the emission shows some similarities with that of GO, such as excitation wavelength and $\mathrm{pH}$ dependent emission. The current results of GO fluorescence could be linked to understand those emissions from rGO and graphene quantum dots. In contrast to the previous studies ${ }^{11,12,16,19,20}$, the present mechanism considers roles of three functionalized groups, sp2 and oxidized sp 3 sites; the $\lambda_{\text {exc }}$ dependent fluorescence and its multiexponential decays were interpreted in terms of both viewpoints of semiconductor electronic band structure and molecular orbital, which agree well with the detailed steady-state and TRF data.

In conclusion, fluorescence of GO in water was studied by use of steady state and time-resolved spectroscopic techniques. We found the excitation wavelength dependent fluorescence and the multiexponential fluorescence decay kinetics at $\lambda_{\mathrm{em}}=500-800 \mathrm{~nm}$. An ultrafast fluorescence $(\sim 1 \mathrm{ps})$ of GO in water was observed at $\lambda_{\mathrm{em}}=540-$ $660 \mathrm{~nm}$ for the first time. The origin of fluorescence is attributed to the electron-hole recombination from the bottom of $\mathrm{CB}$ and neighbor localized states to wide-range VB. The carrier lifetime at the bottom is about $2 \mathrm{~ns}$, which is confirmed by both transient absorption and TRF measurements. Moreover, based on molecular orbital concept, the emission species of predominant visible fluorescence are demonstrated being non-oxidized carbon atom regions and the boundary of oxidized carbon atoms. All three kinds of functionalized groups $\mathrm{C}$ $\mathrm{O}, \mathrm{C}=\mathrm{O}$ and $\mathrm{O}=\mathrm{C}-\mathrm{OH}$ are involved in the fluorescence of $\mathrm{GO}$. 
13. Sun, X. et al. Nano-graphene oxide for cellular imaging and drug delivery. Nano Res 1, 203-212 (2008).

14. Cuong, T. V. et al. Photoluminescence and Raman studies of graphene thin films prepared by reduction of graphene oxide. Mater. Lett. 64, 399-401 (2010).

15. Shukla, S. \& Saxena, S. Spectroscopic investigation of confinement effects on optical properties of graphene oxide. Appl. Phys. Lett. 98, 073104 (2011).

16. Eda, G. et al. Blue photoluminescence from chemically derived graphene oxide. Adv. Mater. 22, 505-509 (2010)

17. Subrahmanyam, K. S., Kumar, P., Nag, A. \& Rao, C. N. R. Blue light emitting graphene-based materials and their use in generating white light. Solid State Commun. 150, 1774-1777 (2010)

18. Xin, G. et al. Tunable photoluminescence of graphene oxide from near-ultraviolet to blue. Mater. Lett. 74, 71-73 (2012).

19. Galande, C. et al. Quasi-molecular fluorescence from graphene oxide. Sci. Rep. 1, 85 (2011).

20. Gokus, T. et al. Making graphene luminescent by oxygen plasma treatment. ACS Nano 3(12), 3963-3968 (2009).

21. Paredes, J. I., Villar-Rodil, S., Martinez-Alonso, A. \& Tascon, J. M. D. Graphene oxide dispersions in organic solvents. Langmuir 24, 10560-10564 (2008).

22. Kolmakov, A. et al. Graphene oxide windows for in situ environmental cell photoelectron spectroscopy. Nat. Nanotechnol. 6, 651-657 (2011).

spectroscopy (Thermo Fisher Scientific, UK) was used to determine the element contents. X-ray powder diffraction measurements were carried out by use of a Bruker D8 Advance diffractometer with $\mathrm{Cu} \mathrm{K} \alpha$ radiation. A Raman system (CRM 200, WITec) with a $532 \mathrm{~nm}$ excitation source of diode-pumped laser was employed to measure Raman spectra. Steady state absorption spectra between 200 and $900 \mathrm{~nm}$ were detected by a UV-Vis spectrophotometer (Cary 100 Bio, Varian) with $1.0 \mathrm{~nm}$ spectral resolution. The NIR absorption spectrum from 900 to $2450 \mathrm{~nm}$ was taken by a UV/Vis/NIR Spectrometer (PerkimElmer Lambda 950). A spectrofluorometer (Fluorolog-3, HORIBA Jobin Yvon) was used to record the steady state fluorescence excitation and emission spectra.

Transient absorption spectra were taken by ultrafast pump-probe system, which has been described in the previous publications ${ }^{50,51}$. The pump wavelength of $480 \mathrm{~nm}$ was chosen and the white light continuum was used as the probe beam. Time-resolved fluorescence (TRF) spectra were measured at room temperature by use of timecorrelated single photon counting (TCSPC) technique with resolution of $10 \mathrm{ps}$ (PicoQuant PicoHarp 300). For TRF, the second harmonic laser beams of Titanium sapphire laser (Chameleon, Coherent Inc.) at 400 and $480 \mathrm{~nm}(100 \mathrm{fs}, 80 \mathrm{MHz})$ were utilized as the excitation source ${ }^{52}$. For TCSPC measurements, the instrument response function is about $30 \mathrm{ps}$. After deconvolution fitting, the temporal resolution is $\sim 10$ ps. A femtosecond fluorescence up-conversion Spectrometer (FOG100, CDP) was used to measure the ultrafast fluorescence decay signals with $400 \mathrm{~nm}$ excitation femtosecond laser source (100 fs, $80 \mathrm{MHz}$ ). Absorption/TCPSC and upconversion/ pump-probe measurements were performed in $1 \mathrm{~cm}$ and $2 \mathrm{~mm}$ quartz cuvettes, respectively. For the fluorescence upconversion case, a flow cuvette was used.

DFT calculations. The model of GO was built according to the experimental XPS data, which gave $\mathrm{C}: \mathrm{O}=2.4: 1$, and where $5 * 6$ super-cell (including 60 carbon atoms) was used. Density functional theory (DFT/GGA-PBE) was implemented to calculate the molecular orbital, band structure, density of states in Material studio $5.5 \mathrm{Dmol} 3$ module with the DNP 4.4 basis including TS correction ${ }^{53}$. Core electron was treated by All Electron. All the parameters are fine. Beside, the absorption spectrum was calculated in Castep module using the same method, where the pseudopotentials are norm-conserving and the energy cut off is $750 \mathrm{eV}$.

1. Novoselov, K. S. et al. Two-dimensional gas of massless Dirac fermions in graphene. Nature 438, 197-200 (2005).

2. Geim, A. K. Graphene: Status and prospects. Science 324(5934), 1530-1534 (2009).

3. Novoselov, K. S. Graphene: Materials in the flatland. Rev. Mod. Phys. 83, 837-849 (2011)

4. Dikin, D. A. et al. Preparation and characterization of graphene oxide paper. Nature 448, 457-460 (2007).

5. Dreyer, D. R., Park, S., Bielawski, C. W. \& Ruoff, R. S. The chemistry of graphene oxide. Chem. Soc. Rev. 39, 228-240 (2010).

6. Eda, G. \& Chhowalla, M. Chemically derived graphene oxide: towards large-area thin-film electronics and optoelectronics. Adv. Mater. 22, 2392-2415 (2010).

7. Nair, R. R., Wu, H. A., Jayaram, P. N., Grigorieva, I. V. \& Geim, A. K. Unimpeded permeation of water through helium-leak-tight graphene-based membranes. Science 335, 442-444 (2012).

8. Stoller, M. D., Park, S., Zhu, Y., An, J. \& Ruoff, R. S. Graphene-based ultracapacitors. Nano Lett. 8(10), 3498-3502 (2008).

9. Eda, G., Fanchini, G. \& Chhowalla, M. Large-area ultrathin films of reduced graphene oxide as a transparent and flexible electronic material. Nat. Nanotechnol. 3, 270-274 (2008).

10. Loh, K. P., Bao, Q., Eda, G. \& Chhowalla, M. Graphene oxide as a chemically tunable platform for optical applications. Nat. Chem. 2, 1015-1024 (2010).

11. Chien, C.-T. et al. Tunable photoluminescence from graphene oxide. Angew. Chem. Int. Ed. 51, 1-6 (2012).

12. Luo, Z., Vora, P. M., Mele, E. J., Johnson, A. T. C. \& Kikkawa, J. M. Photoluminescence and band gap modulation in graphene oxide. Appl. Phys. Lett. 94, 111909 (2009).
23. Marcano, D. C. et al. Improved synthesis of graphene oxide. ACS Nano 4(8), 4806-4814 (2010).

24. Jeong, H. Y. et al. Graphene Oxide Thin films for flexible nonvolatile memory applications. Nano Lett. 10, 4381-4386 (2010).

25. Kudin, K. N. et al. Raman spectra of graphite oxide and functionalized graphene sheets. Nano Lett. 8(1), 36-41 (2008).

26. Mattevi, C. et al. Evolution of electrical, chemical, and structural properties of transparent and conducting chemically derived graphene thin films. Adv. Funct. Mater. 19, 2577-2583 (2009).

27. Luo, Z., Lu, Y., Somers, L. A. \& Johnson, A. T. C. High yield preparation of macroscopic graphene oxide membranes. J. Am. Chem. Soc. 131, 898-899 (2009)

28. Langford, V. S., McKinley, A. J. \& Quickenden, T. I. Temperature dependence of the visible-near-infrared absorption spectrum of liquid water. J. Phys. Chem. A 105, 8916-8921 (2001).

29. Liu, Z.-B. et al. Ultrafast dynamics and nonlinear optical responses from $\mathrm{sp2}$ - and sp3-hybridized domains in graphene oxide. J. Phys. Chem. Lett. 2, 1972-1977 (2011).

30. Kaniyankandy, S., Achary, S. N., Rawalekar, S. \& Ghosh, H. N. Ultrafast relaxation dynamics in graphene oxide: Evidence of electron trapping. J. Phys. Chem. C 115, 19110-19116 (2011).

31. Subach, F. V. \& Verkhusha, V. V. Chromophore transformations in red fluorescent proteins. Chem. Rev. DOI: 10.1021/cr2001965, in press (2012).

32. Yan, J.-A., Xian, L. \& Chou, M. Y. Structural and electronic properties of oxidized graphene. Phys. Rev. Lett. 103, 086802 (2009).

33. Yan, J. A. \& Chou, M. Y. Oxidation functional groups on graphene: Structural and electronic properties. Phys. Rev. B 82, 125403 (2010).

34. Pandey, D., Reifenberger, R. \& Piner, R. Scanning probe microscopy study of exfoliated oxidized graphene sheets. Surf. Sci. 602, 1607-1613 (2008).

35. Bansal, T. et al. New insights into the density of states of graphene oxide using capacitive photocurrent spectroscopy. Carbon 50, 808-814 (2012).

36. Anderson, P. W. Absence of diffusion in certain random lattices. Phys. Rev. 109, 1492-1505 (1958).

37. Evers, F. \& Mirlin, A. D. Anderson transitions. Rev. Mod. Phys. 80, 1355-1417 (2008).

38. Das Sarma, S., Adam, S., Hwang, E. H. \& Rossi, E. Electronic transport in twodimensional graphene. Rev. Mod. Phys. 83, 407-470 (2011).

39. Pereira, V. M., Guinea, F., Lopes dos Santos, J. M. B., Peres, N. M. R. \& Neto, A. H. C. Disorder induced localized states in graphene. Phys. Rev. Lett. 96, 036801 (2006).

40. Leconte, N. et al. Quantum transport in chemically modified two-dimensional graphene: From minimal conductivity to Anderson localization. Phys. Rev. B 84, 235420 (2011).

41. Martin, I. \& Blanter, Y. M. Transport in disordered graphene nanoribbons. Phys.

42. Schubert, G. \& Fehske, H. Metal-to-insulator transition and electron-hole puddle formation in disordered graphene nanoribbons. Phys. Rev. Lett. 108, 066402 (2012).

43. Itoh, T. Fluorescence and phosphorescence from higher excited states of organic molecules. Chem. Rev. DOI: 10.1021/cr200166m, in press, (2012).

44. Pan, D., Zhang, J., Li, Z. \& Wu, M. Hydrothermal route for cutting graphene sheets into blue-luminescent graphene quantum dots. Adv. Mater. 22, 734-738 (2010).

45. Peng, J. et al. Graphene quantum dots derived from carbon fibers. Nano Lett. 12, 844-849 (2012).

46. Zhu, S. et al. Graphene quantum dots with controllable surface oxidation, tunable fluorescence and up-conversion emission. RSC Advances 2, 2717-2720 (2012).

47. Hummers, Jr., W. S. \& Offeman, R. E. Preparation of graphitic oxide. J. Am. Chem. Soc. 80(6), 1339-1339 (1958).

48. Cote, L. J., Kim, F. \& Huang, J. Langmuir-blodgett assembly of graphite oxide single layers. J. Am. Chem. Soc. 131, 1043-1049 (2009).

49. Zhou, X. \& Liu, Z. A scalable, solution-phase processing route to graphene oxide and graphene ultralarge sheets. Chem. Commun. 46, 2611-2613 (2010). Rev. B 79, 235132 (2009) 
50. Shang, J., Yu, T., Lin, J. \& Gurzadyan, G. G. Ultrafast electron-optical phonon scattering and quasiparticle lifetime in CVD-grown graphene. ACS Nano 5(4), 3278-3283 (2011)

51. Shang, J., Yu, T. \& Gurzadyan, G. G. Femtosecond energy relaxation in suspended graphene: Phonon-assisted spreading of quasiparticle distribution. Appl. Phys. B 107, 131-136 (2012).

52. Ma, L. et al. Singlet fission in rubrene single crystal: direct observation by femtosecond pump-probe spectroscopy. Phys. Chem. Chem. Phys. 14, 8307-8312 (2012).

53. Tkatchenko, A. \& Scheffler, M. Accurate molecular Van Der Waals interactions from ground-state electron density and free-atom reference data. Phys. Rev. Lett. 102, 073005 (2009).

\section{Acknowledgements}

We are grateful to Professor Maria-Elisabeth Michel-Beyerle for continuous support. We thank Prof. Qihua Xiong and Mr. Dehui Li for near infrared absorption measurements. Shang thanks Dr. Zhiqiang Luo, Dr. Rui Chen, Mr. Jiaxu Yan, Mr. Mingjie Li, Mr. Jun Yin, Mr. Jilei Liu, Ms. Linfei Lai and Dr. Xiangyang Wu for their discussions. Yu thanks the support of the Singapore National Research Foundation under NRF Award No. NRF-RF2010-07 and MOE Tier 2 MOE2009-T2-1-037.

\section{Author contributions}

JS, LM, TY and GGG conceived the experiments; JS, LM and GGG performed the experiments; JS and WA were involved in the sample preparation; JL performed the DFT calculations; JS, TY and GGG prepared the manuscript.

\section{Additional information}

Supplementary information accompanies this paper at http://www.nature.com/ scientificreports

Competing financial interests: The authors declare no competing financial interests.

License: This work is licensed under a Creative Commons

Attribution-NonCommercial-NoDerivative Works 3.0 Unported License. To view a copy of this license, visit http://creativecommons.org/licenses/by-nc-nd/3.0/

How to cite this article: Shang, J. et al. The Origin of Fluorescence from Graphene Oxide. Sci. Rep. 2, 792; DOI:10.1038/srep00792 (2012). 